\title{
PEEK versus titanium cages in lateral lumbar interbody fusion: a comparative analysis of subsidence
}

\author{
Peter G. Campbell, MD, David A. Cavanaugh, MD, Pierce Nunley, MD, Philip A. Utter, MD, \\ Eubulus Kerr, MD, Rishi Wadhwa, MD, and Marcus Stone, PhD \\ Spine Institute of Louisiana, Shreveport, Louisiana
}

OBJECTIVE The authors have provided a review of radiographic subsidence after lateral lumbar interbody fusion (LLIF) as a comparative analysis between titanium and polyetheretherketone (PEEK) cages. Many authors describe a reluctance to use titanium cages in spinal fusion secondary to subsidence concerns due to the increased modulus of elasticity of metal cages. The authors intend for this report to provide observational data regarding the juxtaposition of these two materials in the LLIF domain.

METHODS A retrospective review of a prospectively maintained database identified 113 consecutive patients undergoing lateral fusion for degenerative indications from January to December 2017. The surgeons performing the cage implantations were two orthopedic spine surgeons and two neurosurgeons. Plain standing radiographs were obtained at 1-2 weeks, 8-12 weeks, and 12 months postoperatively. Using a validated grading system, interbody subsidence into the endplates was graded at these time points on a scale of 0 to III. The primary outcome measure was subsidence between the two groups. Secondary outcomes were analyzed as well.

RESULTS Of the 113 patients in the sample, groups receiving PEEK and titanium implants were closely matched at 57 and 56 patients, respectively. Cumulatively, 156 cages were inserted and recombinant human bone morphogenetic protein-2 (rhBMP-2) was used in 38.1\%. The average patient age was 60.4 years and average follow-up was 75.1 weeks. Subsidence in the titanium group in this study was less common than in the PEEK cage group. At early follow-up, groups had similar subsidence outcomes. Statistical significance was reached at the 8- to 12-week and 52-week follow-ups, demonstrating more subsidence in the PEEK cage group than the titanium cage group. rhBMP-2 usage was also highly correlated with higher subsidence rates at all 3 follow-up time points. Age was correlated with higher subsidence rates in univariate and multivariate analysis.

CONCLUSIONS Titanium cages were associated with lower subsidence rates than PEEK cages in this investigation. Usage of rhBMP-2 was also robustly associated with higher endplate subsidence. Each additional year of age correlated with an increased subsidence risk. Subsidence in LLIF is likely a response to a myriad of factors that include but are certainly not limited to cage material. Hence, the avoidance of titanium interbody implants secondary solely to concerns over a modulus of elasticity likely overlooks other variables of equal or greater importance.

https://thejns.org/doi/abs/10.3171/2020.6.FOCUS20367

KEYWORDS XLIF; LLIF; DLIF; direct; extreme; lateral lumbar interbody fusion; subsidence; PEEK; titanium; polyetheretherketone; comparative effectiveness

\section{$\mathrm{L}$} ATERAL lumbar interbody fusion (LLIF) and oblique lateral interbody fusion (OLIF) procedures are a retroperitoneal approach to gain access to the lumbar spine for the purposes of disc resection, endplate preparation, graft implantation, and fusion. These approaches have grown in popularity as less invasive alternatives to isolated direct anterior and posterior access channels. Benefits of the lateral approach include preservation of the anterior longitudinal ligament maintaining segmental stability, significant muscle sparing, fewer abdominal visceral and vascular injuries, and fewer postoperative wound infections. ${ }^{1-3}$

The central tenet of lateral surgery involves placement of an interbody cage between two preserved vertebral body endplates that allows for restoration of height, direct and indirect decompression of neural elements, restoration of the anterior column weight-bearing function, and

ABBREVIATIONS LLIF = lateral lumbar interbody fusion; OLIF = oblique lateral interbody fusion; PEEK = polyetheretherketone; PLIF = posterior lumbar interbody fusion; rhBMP-2= recombinant human bone morphogenetic protein-2.

SUBMITTED May 1, 2020. ACCEPTED June 18, 2020.

INCLUDE WHEN CITING DOI: 10.3171/2020.6.FOCUS20367. 
correction of focal and segmental spinal deformities. ${ }^{4}$ Accordingly, loss of height postoperatively can lead to graft subsidence into the endplates, an undesirable complication. Subsidence is of particular concern for patients undergoing LLIF, secondary to the procedural reliance upon indirect decompression. ${ }^{5}$ Cage subsidence also may allow for decreased segmental stability, loss of disc space height, and loss of foraminal height, which also may ultimately increase recurrence of symptoms and potentially the need for additional surgical procedures. ${ }^{6}$

In the evolution of spinal surgery, the late 1990s saw polyetheretherketone (PEEK) begin to be frequently utilized in spinal fusions due primarily to two proposed attributes of the material. The minimal radiographic interference of a PEEK device on plain radiographs and CT provides a benefit to postoperative evaluation, with less interference than its in-class competitors. PEEK also possesses a modulus of elasticity closer to that of cortical bone than titanium or composites, leading many authors to posit lower stress shielding, less bone remodeling, and decreased subsidence with PEEK usage as compared to titanium. ${ }^{7,8}$ However, PEEK is a hydrophobic polymer that disallows material integration into bone and may be associated with cage migration and pseudoarthrosis. ${ }^{9}$ The bone-to-implant interface has been described as the "PEEK-halo effect" as gaps have been identified between bones and implant material due to poor surface osseointegration. ${ }^{10}$ Another popular material for interbody cages is titanium and its associated alloys. Titanium alloys possess more strength under physiological loads and are likely more osseoconductive and bioactive., ${ }^{41}$ Some authors propose that newer-generation alterations to titanium implant surfaces may ultimately increase bone formation, enhance osseous integration, and improve implant stability in interbody spinal fusions compared with the previous-generation smooth titanium and their PEEK counterparts. ${ }^{12}$

The aim of this review is to provide a comparative analysis between potential variance in cage subsidence as it relates to materials in LLIF. To understand these differences, we reviewed our institutional experience with lateral lumbar interbody cage placement in a private orthopedic and neurosurgical spine practice and present for review the resultant radiographic subsidence outcomes.

\section{Methods \\ Study Population}

An IRB-approved retrospective review of a prospectively collected database was performed at a single spine center with patients from two orthopedic spine and two neurological surgeons. The authors collected data on 114 consecutive patients who underwent LLIF performed between January 1, 2017, and December 31, 2017. Patients undergoing lateral surgery from L1 to S1 were included. One patient was excluded as he did not follow up after hospital discharge due to a military commitment. All patients with degenerative pathology were included. Patients with infectious and neoplastic indications were excluded. Surgical indications most commonly included spondylolisthesis, adult degenerative scoliosis, adjacent-segment pathology, and degenerative disc disease.

\section{Data Collection}

Records from the included patients were evaluated. All patients underwent preoperative 4-view lumbar radiographs and MRI studies. All patients underwent standing 2-view lumbar imaging within 1-2 weeks of surgical intervention. These data were used as the baseline subsidence. Data from standing 2- and 4-view lumbar radiographs obtained between 2-3 months and at or after the 12-month follow-up were also included. If CT data were available at the 1-year time point, they were reviewed to validate the subsidence grading. Both dual-energy x-ray absorptiometry results and surgeon description of poor bone quality intraoperatively were collected for analysis. Prior or current history of tobacco abuse was also reported as an independent variable. Cage size in width, height, and length was recovered from the operative implant log. The use of recombinant human bone morphogenetic protein-2 (rhBMP-2) was also identified.

Subsidence was graded based upon the Marchi classification. ${ }^{13}$ After obtaining standing lateral radiographs and parallel endplates at the index level, subsidence was quantified based on the amount of cage violation into the vertebral endplates. In this classification scheme, grade 0 correlated with $0 \%-24 \%$, grade I $25 \%-49 \%$, grade II $50 \%-74 \%$, and grade III $75 \%-100 \%$ endplate subsidence. Grades 0 and I are considered low-grade subsidence, while grades II and III are considered high-grade subsidence. ${ }^{13}$ Standing lateral radiographs were assessed in the initial phase (1-2 weeks), at the early follow-up (8-12 weeks), and again at the late phase (12-24 months). In patients with a significant coronal imbalance that made obtaining parallel endplates on plain radiographs difficult, CT was also obtained at 12 months to confirm the degree of subsidence.

\section{Surgical Technique}

A lateral technique was performed according to surgeon preference. Within the sample, 112 underwent transpsoas interventions and 1 patient underwent an anterior to psoas approach to the lateral spine with a lateral cage placement. All endplates were prepared for fusion in an attempt to preserve the osseous structure of the endplate. Cartilaginous endplates were removed with a variety of surgeon-specific techniques, typically rasps and shavers. All patients underwent concomitant posterior lumbar arthrodesis with pedicle screw fixation in addition to the lateral cage.

Bone graft materials included in the cage were either demineralized allograft fibers, rhBMP-2, or both. Cages from 5 manufacturers were implanted in the patient cohort. Within those interbody footprints, cage width ranged from 18 to $26 \mathrm{~mm}$. Cage height ranged from 8 to $14 \mathrm{~mm}$. Cage lengths ranged from 45 to $60 \mathrm{~mm}$. Cage lordosis ranged from $7^{\circ}$ to $15^{\circ}$. Figure 1 demonstrates the appearance on postoperative CT of the only two types of titanium cage designs implanted in the study.

\section{Statistical Analysis}

Subsidence grades were compared at 2 weeks, 3 months, and 12 months postsurgery. Univariate analyses were 


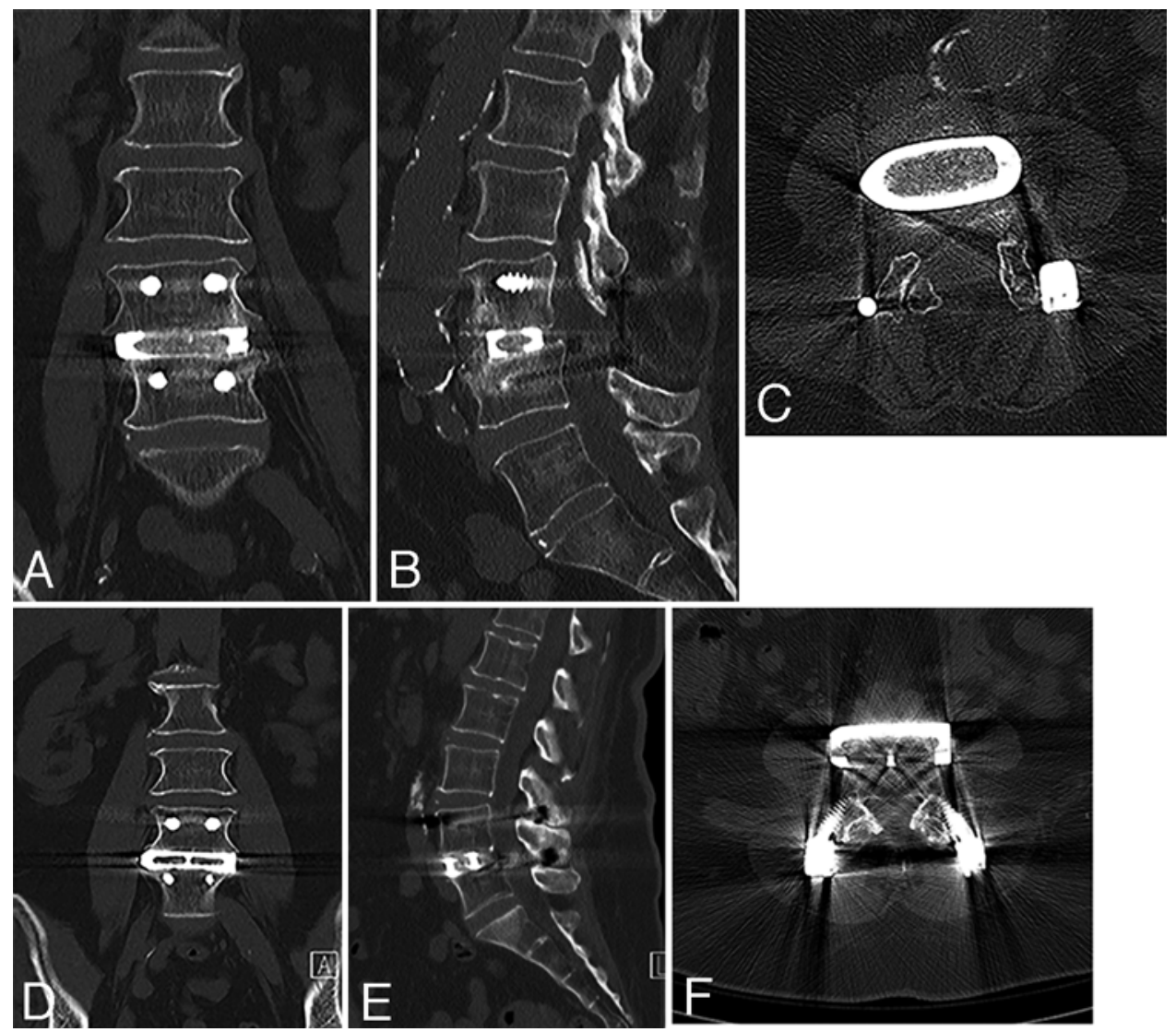

FIG. 1. Coronal ( $A$ and D), sagittal ( $B$ and $E$ ), and axial ( $C$ and $F)$ CT images show the first $(A-C)$ and second (D-F) type of titanium implant used in the study.

conducted to assess the association between subsidence outcome and cage material, operating surgeon, smoking status, osteopenia and osteoporosis, rhBMP-2 usage, and patient age. The Wilcoxon rank-sum test was used to compare the distribution of subsidence grades across strata. Fisher's exact test was used to compare a binarized subsidence outcome (grade 0 vs grades I, II, and III).

Multivariable logistic regression was performed using a binarized subsidence outcome. Cage material, osteopenia/ osteoporosis, rhBMP-2 use, and patient age were included as covariates. A second set of logistic regression models were fit to compare cage materials. Binarized subsidence was used as the outcome. Bivariate models were fit with cage material and either osteopenia and/or osteoporosis, rhBMP-2 use, or patient age, along with an interaction term. Wald tests were used to test for significance of the odds ratio (OR) parameter estimates. A p value $\leq 0.05$ was considered statistically significant. No multiplicity adjustments were performed.

\section{Results}

The study included 113 patients. Table 1 depicts the initial demographic and treatment data. The average age at the time of surgery was 60.4 years. Males made up $38.9 \%(n=44)$ of the study participants and females accounted for $61.1 \%(n=69)$ of the entire study. Patient
TABLE 1. Baseline demographic and treatment data

\begin{tabular}{lc}
\hline \multicolumn{1}{c}{ Variable } & Value \\
\hline No. of patients & 113 \\
\hline No. of levels & 156 \\
\hline Mean age in yrs \pm SD & $60.4 \pm 12.3$ \\
\hline Males, $\mathrm{n}(\%)$ & $44(38.9)$ \\
\hline Females, $\mathrm{n}(\%)$ & $69(61.1)$ \\
\hline PEEK by patient & 57 \\
\hline Titanium by patient & 56 \\
\hline Smokers, $\mathrm{n}(\%)$ & $24(21.2)$ \\
\hline rhBMP-2 usage, $\mathrm{n}(\%)$ & $43(38.1)$ \\
\hline Levels treated, $\mathrm{n}(\%)$ & \\
\hline 1 & $74(65.5)$ \\
\hline 2 & $35(31.0)$ \\
\hline 3 & $4(3.5)$ \\
\hline Posterior instrumentation & $100 \%$ \\
\hline Cage width in mm, $\%$ implanted & \\
\hline 18 & 12.0 \\
\hline 22 & 86.6 \\
\hline 26 & 1.4 \\
\hline Mean follow-up in wks \pm SD & $75.1 \pm 45.5$ \\
\hline
\end{tabular}


TABLE 2. Demographic and treatment data group comparison

\begin{tabular}{lccl}
\hline \multicolumn{1}{c}{ Variable } & Titanium & PEEK & p Value \\
\hline No. of patients & 56 & 57 & \\
\hline No. of levels & 72 & 84 & 0.20 \\
\hline Mean age in yrs & 61.8 & 59.0 & 0.39 \\
\hline Males & 22 & 22 & 1 \\
\hline Females & 34 & 35 & \\
\hline Smokers, $\mathrm{n}(\%)$ & $7(12.5)$ & $17(29.8)$ & 0.04 \\
\hline rhBMP-2 usage, $\mathrm{n}(\%)$ & $0(0)$ & $42(73.7)$ & 0.0001 \\
\hline Osteopenia/osteoporosis, $\mathrm{n}(\%)$ & $9(16.1)$ & $13(22.8)$ & 0.48 \\
\hline Cage width in mm & & & \\
\hline 18 & $19.4 \%$ & $11.9 \%$ & \\
\hline 22 & $80.6 \%$ & $85.7 \%$ & 0.01 \\
\hline 26 & 0 & $2.4 \%$ & \\
\hline Mean follow-up in wks & 77.2 & 73.1 & \\
\hline Lost to 12-month follow-up & 12 & 9 & \\
\hline
\end{tabular}

groups receiving PEEK and titanium implants were closely matched at 57 and 56 patients, respectively. A total of 156 levels were fused. The most common indication for surgery in this sample was an adjacent-segment revision to a previous fusion $(34.5 \%, \mathrm{n}=39)$. All patients undergoing multiple-level fusions had only 1 cage material implanted. rhBMP-2 was used in $38.1 \%$ of the cases $(n=43)$. Smokers made up $21.2 \%$ of the sample. Osteopenia was present in $12.4 \%$ of the sample and osteoporosis was documented in
$6.2 \%$ of the patients preoperatively. Baseline demographic and treatment group comparisons are available in Table 2.

Groups were quite closely matched in terms of group size, age, sex, and length of follow-up. None of these variables were significantly different. Tobacco usage was reported in a higher proportion of patients undergoing PEEK as compared to titanium implants at $29.8 \%$ and $12.5 \%$, respectively $(\mathrm{p}=0.037)$. Osteopenia and osteoporosis were slightly more common in the PEEK group, but this difference did not reach statistical significance $(p=0.476)$. Cage widths of $18 \mathrm{~mm}$ were more frequent in the titanium cage group, while 22 and $26 \mathrm{~mm}$ were more frequently utilized in the PEEK cage group $(\mathrm{p}=0.01)$. Twelve titanium cage and 9 PEEK cage patients were lost to 12-month follow-up (Table 2).

The average follow-up duration was 75.1 weeks. Three patients did not follow up after the initial 2-week followup. Twenty-one patients did not have 12-month follow-up. Given that subsidence is not an improving condition, if patients were noted to have been determined to have experienced subsidence at any time point, but were lost to subsequent follow-up, they were included as the last grade of reported subsidence for the purposes of analysis. Hence, these patients were included in final analysis under the above parameters.

The overall incidence of subsidence of the PEEK and titanium cages is depicted graphically in Figs. 2 and 3, respectively. In the titanium group, grade 0 subsidence at initial evaluation was $96.4 \%$ and grade I subsidence was $3.6 \%$. There were no cases of grade II or III subsidence at



FIG. 2. Bar graph of the incidence of subsidence PEEK cage implantation by Marchi grade at three postoperative time points. The $y$-axis represents the number of patients. 


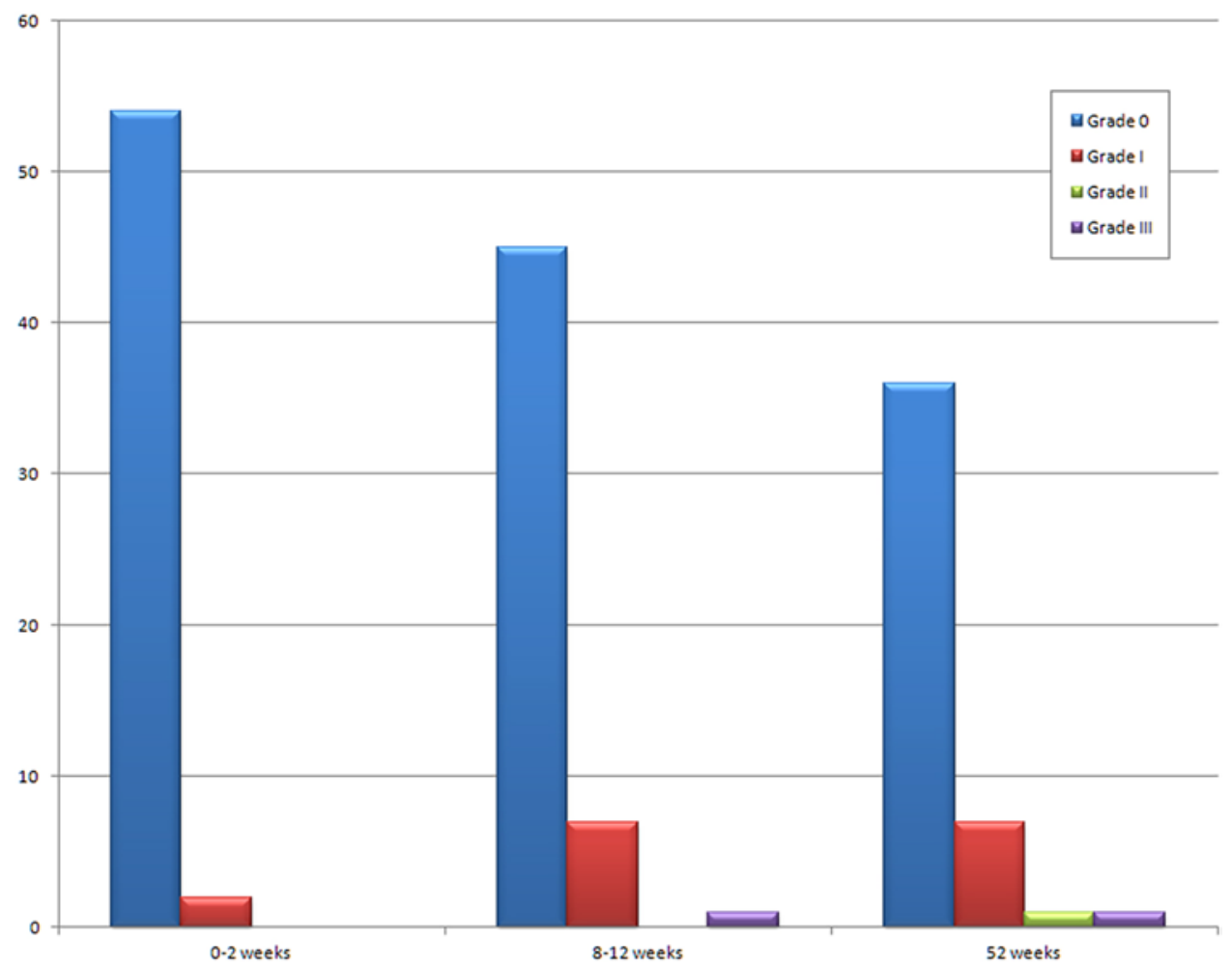

FIG. 3. Bar graph of the incidence of subsidence after titanium cage implantation by Marchi grade at three postoperative time points. The y-axis represents the number of patients.

this initial time point. At 1-year follow-up, $80 \%$ maintained a grade 0 subsidence. Grade I subsidence was identified in $15.6 \%$ at 1 year. Grades II and III were noted in $2.2 \%$ of the sample. In the PEEK group, grade 0 subsidence at initial evaluation was $87.7 \%$ and grade I subsidence was $12.3 \%$. There were no cases of grade II or III subsidence at this time point. At 1-year follow-up 57.1\% maintained a grade 0 subsidence. Grade I subsidence was identified in $22.4 \%$, and grades II and III were seen in $16.3 \%$ and $4.1 \%$ of the sample at 1 year, respectively.

In the statistical analysis, PEEK had a significantly higher subsidence at the 8- to 12 -week interval as well as the 12-month follow-up with $\mathrm{p}$ values of 0.021 and 0.012 , respectively. Titanium trended toward lower subsidence even at initial follow-up, but this did not reach statistical significance $(p=0.090)$. Other variables tested independent of cage material in univariate testing noted to reach statistical significance with respect to increased subsidence are decreased bone mineral density, older patient age at time of surgery, and rhBMP-2 usage. At each followup interval the usage of rhBMP-2 was strongly associated with subsidence with $p$ values of $0.009,0.005$, and 0.0236 , respectively (Fig. 4). Multivariate analysis identified age as the only factor that strongly correlates with a binarized subsidence (Table 3). With each additional year of age, this result identified a correlated increase in the risk of subsidence by factors of 1.05 and 1.07 for the 8 - to 12 -week and 12-month periods, respectively.

The contribution of surgeon-specific outcome to cage subsidence was also considered utilizing a generalized
Fisher's exact test. No evidence of surgeon-specific subsidence was identified that reached statistical significance. Additionally, no increased subsidence was observed in the sample as compared to subsidence in nonsmokers.

During the course of follow-up, 12 patients required additional procedures. Five patients underwent extension of fusion for adjacent-segment pathology within up to 3 years of the follow-up period. One patient required removal of posterior hardware for hardware-related pain after fusion had occurred. Three patients returned to the operating room within 3 months for a posterior decompression that had previously been treated with indirect decompression. Two patients underwent surgical fracture secondary to a sacral fracture related to an L5-S1 ALIF performed during the same hospitalization as the LLIF. One patient returned to the operating room at 6 weeks postoperatively for additional decompression and extension of posterior instrumentation secondary to severe subsidence and development of a burst fracture at the operative level.

There was 1 death in the postoperative period. A 67-year-old man underwent a 2-level LLIF with posterior instrumentation for the indication of unstable spondylolisthesis. Grade III subsidence occurred 6 weeks postoperatively; posterior instrumentation was nonsegmental in this case. Every other patient in the study underwent segmental posterior pedicle screw instrumentation. He was returned to the operating room for extension of his hardware and expanded decompression. The patient ultimately died due to medical complications prior to his 6-month follow-up (Fig. 5). Another return to the operating room 


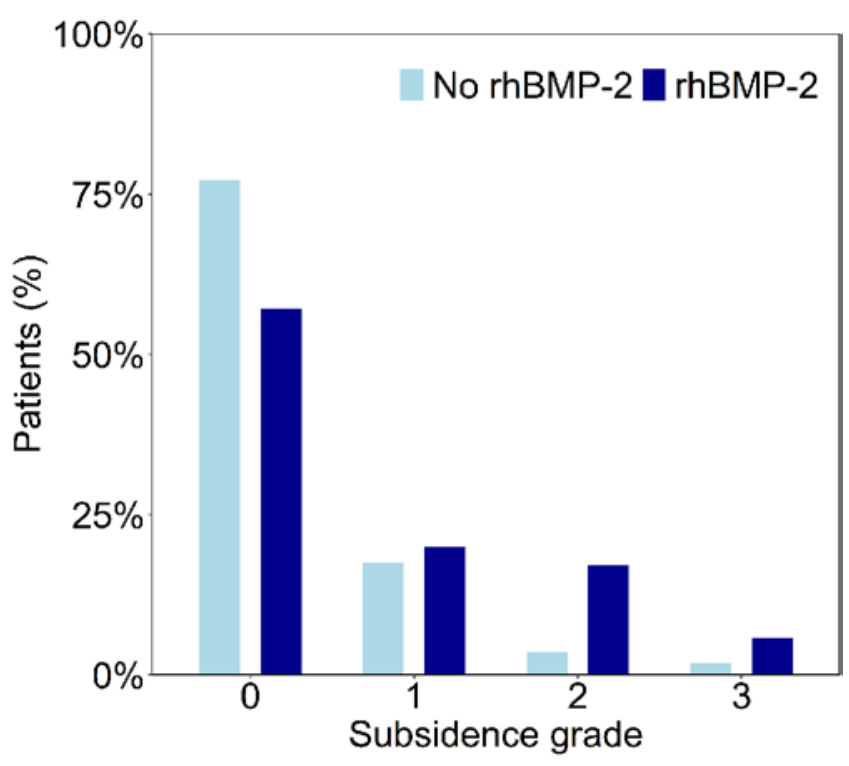

FIG. 4. Bar graph of the relationship between use of rhBMP-2 and increased subsidence by Marchi grade at the 12-month follow-up.

was required in the series also due to severe subsidence. An 82-year-old man with osteopenia underwent a direct lateral L3-4 and an L4-5 anterior to psoas approach with placement of a lateral cage. The L4-5 level could be described as transitional. At 8 weeks of follow-up he was reporting a loss of the improved pain he received initially after surgery. Imaging showed a grade III subsidence at the inferior level. He was taken for a partial facetectomy at that time. He improved clinically and both levels proceeded to successfully fuse at the 3-year time point, as shown on CT (Fig. 6).

\section{Discussion}

A true understanding of interbody materials in spinal surgery requires both an a priori and a posteriori knowledge. In the first type of knowledge, mathematical, physical, and biological information such as implant stiffness, surface integration, and biological compatibility provide background as predictors of outcome. In the second type, the justification for the use of the product relies upon experience and empirical evidence. To the authors' knowledge, this is the first study that attempts to provide a comparative analysis of radiographic subsidence of two matched LLIF populations at a four-surgeon center. This report will seek to add to the a posteriori understanding of material selection for LLIF using our clinical experience.

The volume of lumbar fusion surgeries in the US increased $62.3 \%$ from 2004 to $2015 .{ }^{14}$ Beginning in the early 2000s, a trend toward the usage of spinal implants with a lower modulus of elasticity began in an attempt to match implant stiffness to the bone with which they interface. ${ }^{15}$ PEEK utilization rose during these times, with some authors reporting that a mismatch in the modulus of elasticity between the implanted cage and the host bone may be responsible for the development of subsidence. ${ }^{16}$ While the ultimate stiffness of all biomaterials for spinal implants varies based on manufacturing methods, Heary
TABLE 3. Multivariate analysis of subsidence at 8-12 weeks and 12 months

\begin{tabular}{lrrrr}
\hline \multicolumn{1}{c}{ Follow-Up Interval } & OR & Upper Cl & Lower Cl & p Value \\
\hline 8-12 wks & & & & \\
\hline PEEK usage & 1.45 & 0.28 & 6.21 & 0.626 \\
\hline Osteoporosis or osteopenia & 2.04 & 0.69 & 5.87 & 0.189 \\
\hline rhBMP-2 usage & 2.78 & 0.69 & 14.37 & 0.176 \\
\hline Age & 1.05 & 1.00 & 1.10 & 0.037 \\
\hline 12 mos & & & & \\
\hline PEEK usage & 3.16 & 0.74 & 13.49 & 0.114 \\
\hline Osteoporosis or osteopenia & 2.06 & 0.67 & 6.26 & 0.200 \\
\hline rhBMP-2 usage & 1.39 & 0.34 & 6.05 & 0.6479 \\
\hline Age & 1.07 & 1.02 & 1.12 & 0.011 \\
\hline Cl = confidence interval. & & & &
\end{tabular}

et al. calculated the elastic modulus of pure PEEK and titanium from load displacement curves at 3.84 and 50.20 gigapascals, respectively. ${ }^{16}$ Some authors have expressed concern that a mismatch between the bone and implant of this magnitude could lead to stress shielding and bone remodeling around the implant, thus increasing subsidence rates. ${ }^{7,17}$

However, PEEK implants have several limitations. A PEEK-halo effect has been described, which is thought to result from minimal osseointegration of PEEK at the adjacent vertebral endplate and a biofilm layer around the surface of the impant..$^{10} \mathrm{~A}$ recent review of PEEK in dental implants demonstrated a PEEK inferiority compared with titanium with respect to osseoconductivity and bioactivity of the implant. ${ }^{11}$ Basic science evaluations have demonstrated a superiority of titanium surfaces to PEEK in the achievement of osseointegration, especially if given the appropriate surface topography. ${ }^{18,19}$ Authors have suggested that amorphous coated or altered titanium surfaces stimulate osteogenic protein expression with increased cell-matrix adhesion protein formation important for bone fusion and remodeling. This is reportedly correlated with improvement in early bone growth that leads to significant clinical improvement in later clinical outcomes. ${ }^{20,21}$

While the authors are not aware of comparative studies regarding PEEK versus titanium in the LLIF population, there are similar studies regarding the posterior lumbar interbody fusion (PLIF) technique. In a recent meta-analysis, Massaad et al. extracted all available subsidence data from 6 PLIF comparative studies. ${ }^{22}$ The ranges of subsidence rates in the titanium and PEEK groups for these posterior and posterolateral procedures were $0 \%-36 \%$ and $0 \%-31 \%$, respectively. ${ }^{23-27}$ Based upon the authors' analysis, there was no difference in the rate of subsidence between titanium and PEEK interbody cages in the PLIF population. ${ }^{22}$

Similar extensive comparative evaluations of the literature have been performed in the cervical spine literature as well. A 2016 study included two randomized and two nonrandomized clinical trials comparing PEEK and titanium cage groups. ${ }^{728-30}$ Meta-analysis of these studies revealed an increased subsidence in the titanium group with an OR 


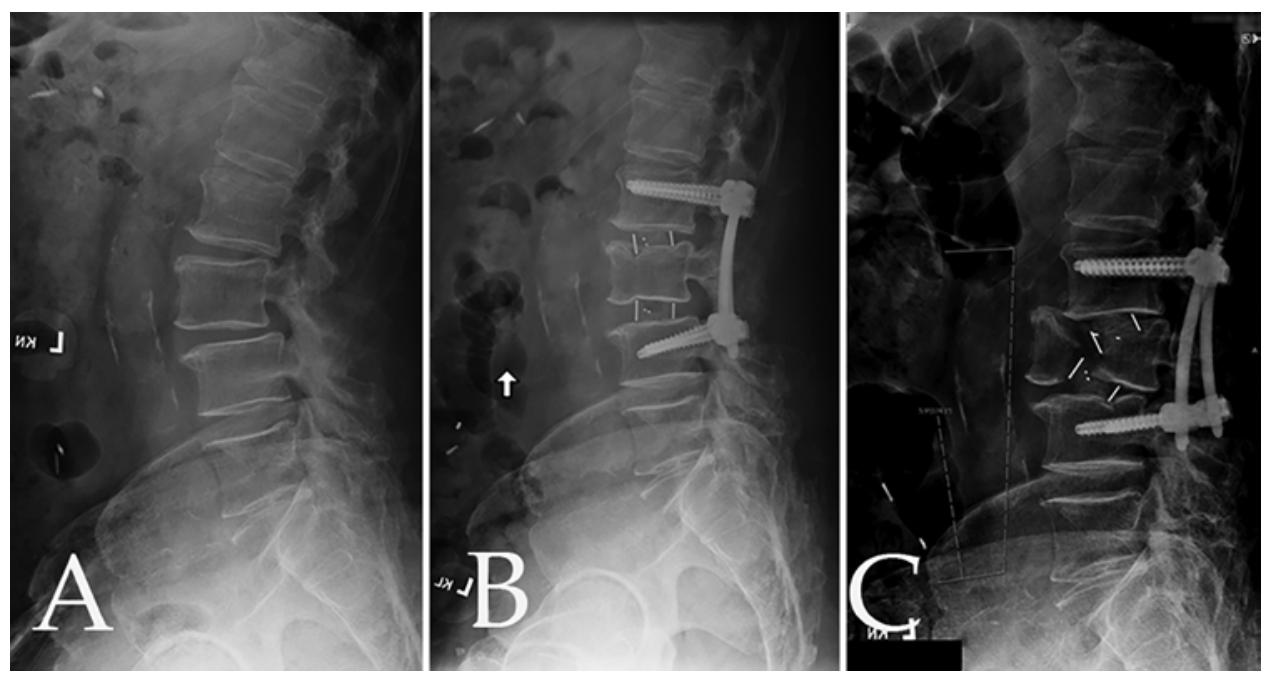

FIG. 5. Standing lateral radiographs. A: Image from a 67-year-old man with grade 1 spondylolisthesis at L3-4. B: Image obtained 1 week after an L2-3 and L3-4 LLIF with posterior instrumentation, showing grade I subsidence. C: Image obtained 6 weeks postoperatively with grade III subsidence at L3.

of 3.14..$^{31}$ This result was believed to describe a more favorable subsidence profile of PEEK in 1- and 2-level anterior cervical fusions as compared to their titanium counterpart.

The incidence of overall subsidence has been reported in the literature. In the early acceptance of lateral interbody surgery, cages provided by the early manufactures were generally PEEK. Hence, most subsidence data are based on the longer-term follow-up of PEEK cages. In 2013, Marchi et al. introduced the subsidence classification system based on plain standing radiographs and PEEK cages. ${ }^{13}$ Additional studies have since utilized and validated this grading scale since its inception. ${ }^{3,32}$ In general, previous studies evaluating LLIF cage subsidence have also found decreasing subsidence with wider implant deployment. ${ }^{3}$ In a study of 140 patients and 238 levels fused with an LLIF technique, Le et al. demonstrated significantly higher rates of subsidence in patients with cages measuring $18 \mathrm{~mm}$ at $14.1 \%$, while those with PEEK cages larger than $22 \mathrm{~mm}$ had a lower subsidence rate at $1.9 \% .{ }^{5}$ Another study investigated the use of even wider cages $(26 \mathrm{~mm})$ and found that these had significantly reduced cage settling compared to both the 18- and 22-mm cages. ${ }^{6}$ Agarwal et al. reported in a standalone LLIF population that the use of $18-\mathrm{mm}$ implants likely increases the risk of developing high-grade subsidence necessitating reoperation when compared to 22-mm-wide implants. ${ }^{3}$

The current study offers the first comparative clinical evaluation of subsidence in PEEK versus titanium lateral interbody cages. There are some limitations to the study parameters. The retrospective nature of the study does not allow for prospective collection of data points. While the groups were closely matched in most baseline characteristics, there were significantly more smokers in the PEEK group. However, wider cages were implanted in the PEEK group as compared to the titanium group. Two types of titanium cages and 3 different PEEK cages were implanted based on surgeon preference, and thus may present cage selection bias. It is also possible that the functional stiff-

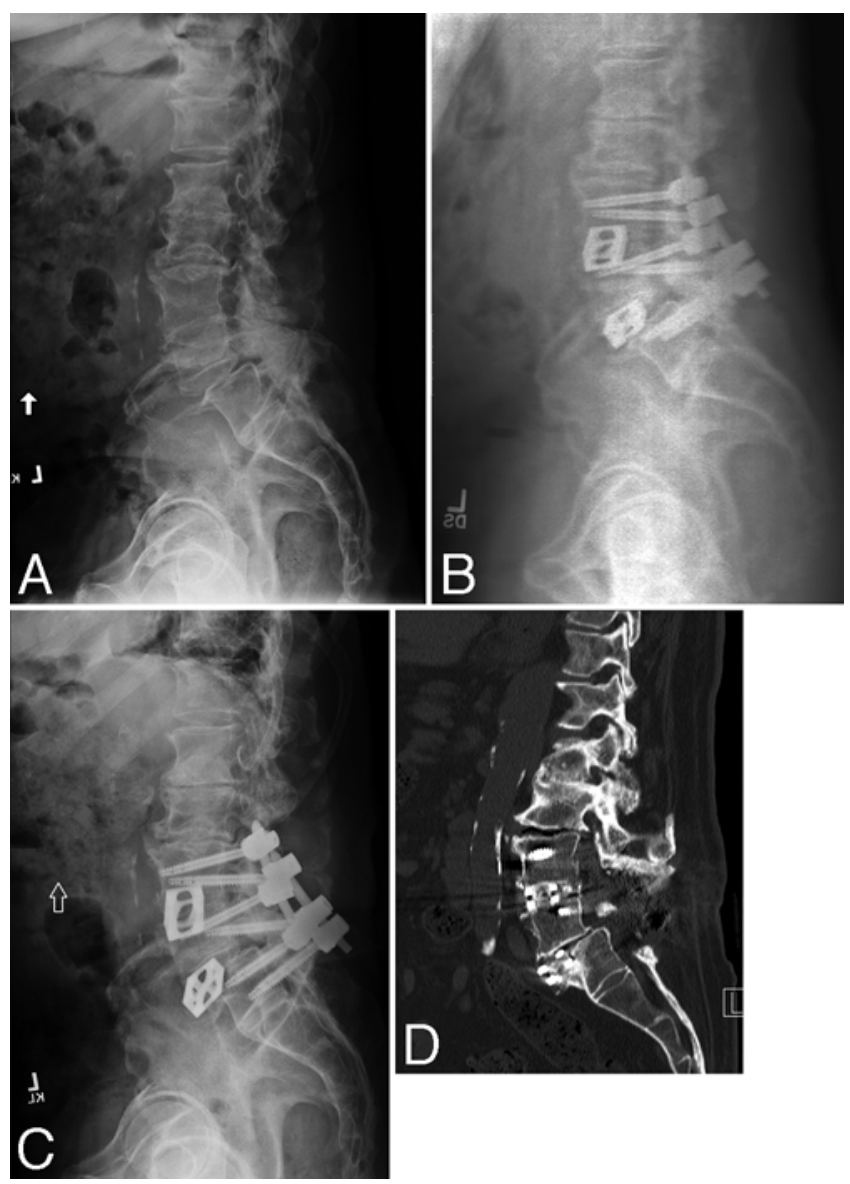

FIG. 6. Standing lateral radiographs (A-C) and CT scan (D). A: Image from an 82-year-old man with grade 1 spondylolisthesis at $L 4-5$ and adult degenerative scoliosis B: One week after an L3-4 and L4-5 LLIF with posterior instrumentation showing grade 0 subsidence C: Six-week postoperative image with grade III subsidence at L5. D: CT scan of the lumbar spine demonstrates stable endplate subsidence into the $L 5$ vertebral body at 3 years. 
ness of the 3D-printed titanium cage made with additive technology differs from the titanium cage manufactured from titanium bar stock with a subtractive manufacturing process. No surgeon implanting titanium cages used any rhBMP-2, which potentially exacerbated the subsidence risk in the PEEK group. Four surgeons-each with their own nuanced techniques for endplate preparation, surgical indications, and risk thresholds-entered patients into the study, possibly imparting some surgeon-specific bias into the results. However, when this particular variable was evaluated statistically, the sample size was likely not large enough to detect a significant difference in outcome. In multivariate analysis, only age was a sufficiently strong factor to produce a statistically significant result. All other variables trended the same way as they did in the univariate analysis, which produced statistically significant results. In this study, the univariate analysis was a rank-based test that evaluated all 4 grades of subsidence. The multivariate analysis used a binary subsidence endpoint. This allows for the possibility that the study lacked significant power at all 4 grades of subsidence to carry these conclusions over to multivariate analysis, due to lack of sample size.

The operative episode required to perform a spinal fusion is a complex series of tasks with no clinical studies providing standardized process mapping of the LLIF procedure. The intraoperative surgical process is undoubtedly influenced by equipment and implant materials, age, patient comorbidities, individual surgeon skill, and case complexity. ${ }^{33}$ For example, if extensive amounts of bone endplates have been excessively removed during endplate preparation for LLIF, then it is likely that even grafts with low modulus values will subside into the damaged endplate. ${ }^{16}$ Additionally, bone density, cage shape, age, and applied distraction may result in increased subsidence rates as well. ${ }^{29}$ Cage width also appears to influence subsidence. However, some authors report the "elimination" of subsidence in LLIF with the utilization of 26-mm cages, which may be an overly simplistic claim. ${ }^{6}$

\section{Conclusions}

While acknowledging its limitations, in the current study titanium was associated with less subsidence than PEEK. Additionally, a strong correlation between rhBMP-2 use and subsidence was noted at each time point studied. Age was the strongest predictor of subsidence. Hence, any study that aims to evaluate the impact of PEEK or titanium cages on subsidence must also consider other essential variables as well. Subsidence in LLIF is most likely the result of a multifactorial process, rather than a binary consequence of cage selection.

\section{Acknowledgments}

We offer our appreciation to the Spine Institute of Louisiana Foundation for administrative support in preparation of this manuscript and to Mr. Kyle Marshall, MS, for assistance with statistical evaluation.

\section{References}

1. Lehmen JA, Gerber EJ. MIS lateral spine surgery: a system- atic literature review of complications, outcomes, and economics. Eur Spine J. 2015;24(suppl 3):287-313.

2. Rajaraman V, Vingan R, Roth P, et al. Visceral and vascular complications resulting from anterior lumbar interbody fusion. J Neurosurg. 1999;91(1)(suppl):60-64.

3. Agarwal N, White MD, Zhang X, et al. Impact of endplateimplant area mismatch on rates and grades of subsidence following stand-alone lateral lumbar interbody fusion: an analysis of 623 levels. J Neurosurg Spine. 2020;33(1):12-16.

4. Willems K, Lauweryns P, Verleye G, VAN Goethem J. Randomized controlled trial of posterior lumbar interbody fusion with Ti- and CaP-nanocoated polyetheretherketone cages: comparative study of the 1-year radiological and clinical outcome. Int J Spine Surg. 2019;13(6):575-587.

5. Le TV, Baaj AA, Dakwar E, et al. Subsidence of polyetheretherketone intervertebral cages in minimally invasive lateral retroperitoneal transpsoas lumbar interbody fusion. Spine (Phila Pa 1976). 2012;37(14):1268-1273.

6. Lang G, Navarro-Ramirez R, Gandevia L, et al. Elimination of subsidence with 26-mm-wide cages in extreme lateral interbody fusion. World Neurosurg. 2017;104:644-652.

7. Niu CC, Liao JC, Chen WJ, Chen LH. Outcomes of interbody fusion cages used in 1 and 2-levels anterior cervical discectomy and fusion: titanium cages versus polyetheretherketone (PEEK) cages. J Spinal Disord Tech. 2010;23(5):310-316.

8. Toth JM, Wang M, Estes BT, et al. Polyetheretherketone as a biomaterial for spinal applications. Biomaterials. 2006;27(3): 324-334.

9. Pelletier MH, Cordaro N, Punjabi VM, et al. PEEK versus Ti interbody fusion devices: resultant fusion, bone apposition, initial and 26-week biomechanics. Clin Spine Surg. 2016; 29(4):E208-E214.

10. Phan K, Hogan JA, Assem Y, Mobbs RJ. PEEK-halo effect in interbody fusion. J Clin Neurosci. 2016;24:138-140.

11. Najeeb S, Khurshid Z, Zohaib S, Zafar MS. Bioactivity and osseointegration of PEEK are inferior to those of titanium: a systematic review. J Oral Implantol. 2016;42(6):512-516.

12. Olivares-Navarrete R, Gittens RA, Schneider JM, et al. Osteoblasts exhibit a more differentiated phenotype and increased bone morphogenetic protein production on titanium alloy substrates than on poly-ether-ether-ketone. Spine $J$. 2012;12(3):265-272.

13. Marchi L, Abdala N, Oliveira L, et al. Radiographic and clinical evaluation of cage subsidence after stand-alone lateral interbody fusion. J Neurosurg Spine. 2013;19(1):110-118.

14. Martin BI, Mirza SK, Spina N, et al. Trends in lumbar fusion procedure rates and associated hospital costs for degenerative spinal diseases in the United States, 2004 to 2015. Spine (Phila Pa 1976). 2019;44(5):369-376.

15. Rousseau MA, Lazennec JY, Saillant G. Circumferential arthrodesis using PEEK cages at the lumbar spine. J Spinal Disord Tech. 2007;20(4):278-281.

16. Heary RF, Parvathreddy N, Sampath S, Agarwal N. Elastic modulus in the selection of interbody implants. J Spine Surg. 2017;3(2):163-167.

17. Peitgen DS, Innmann MM, Merle C, et al. Periprosthetic bone mineral density around uncemented titanium stems in the second and third decade after total hip arthroplasty: a DXA study after 12, 17 and 21 years. Calcif Tissue Int. 2018; 103(4):372-379.

18. Olivares-Navarrete R, Hyzy SL, Gittens RA, et al. Rough titanium alloys regulate osteoblast production of angiogenic factors. Spine J. 2013;13(11):1563-1570.

19. Rao PJ, Pelletier MH, Walsh WR, Mobbs RJ. Spine interbody implants: material selection and modification, functionalization and bioactivation of surfaces to improve osseointegration. Orthop Surg. 2014;6(2):81-89.

20. Arts M, Torensma B, Wolfs J. Porous titanium cervical interbody fusion device in the treatment of degenerative cervical 
radiculopathy; 1-year results of a prospective controlled trial. Spine J. 2020;20(7):1065-1072.

21. Silva-Bermudez P, Almaguer-Flores A, Garcia VI, et al. Enhancing the osteoblastic differentiation through nanoscale surface modifications. J Biomed Mater Res A. 2017;105(2): 498-509.

22. Massaad E, Fatima N, Kiapour A, et al. Polyetheretherketone versus titanium cages for posterior lumbar interbody fusion: meta-analysis and review of the literature. Neurospine. 2020; 17(1):125-135.

23. Cuzzocrea F, Ivone A, Jannelli E, et al. PEEK versus metal cages in posterior lumbar interbody fusion: a clinical and radiological comparative study. Musculoskelet Surg. 2019; 103(3):237-241.

24. Nemoto O, Asazuma T, Yato Y, et al. Comparison of fusion rates following transforaminal lumbar interbody fusion using polyetheretherketone cages or titanium cages with transpedicular instrumentation. Eur Spine J. 2014;23(10):2150-2155.

25. Rickert M, Fleege C, Tarhan T, et al. Transforaminal lumbar interbody fusion using polyetheretherketone oblique cages with and without a titanium coating: a randomised clinical pilot study. Bone Joint J. 2017;99-B(10):1366-1372.

26. Sakaura H, Ohnishi A, Yamagishi A, Ohwada T. Early fusion status after posterior lumbar interbody fusion with cortical bone trajectory screw fixation: a comparison of titaniumcoated polyetheretherketone cages and carbon polyetheretherketone cages. Asian Spine J. 2019;13(2):248-253.

27. Wrangel CV, Karakoyun A, Buchholz KM, et al. Fusion rates of intervertebral polyetheretherketone and titanium cages without bone grafting in posterior interbody lumbar fusion surgery for degenerative lumbar instability. J Neurol Surg A Cent Eur Neurosurg. 2017;78(6):556-560.

28. Chen Y, Wang X, Lu X, et al. Comparison of titanium and polyetheretherketone (PEEK) cages in the surgical treatment of multilevel cervical spondylotic myelopathy: a prospective, randomized, control study with over 7-year follow-up. Eur Spine J. 2013;22(7):1539-1546.

29. Cabraja M, Oezdemir S, Koeppen D, Kroppenstedt S. Anterior cervical discectomy and fusion: comparison of titanium and polyetheretherketone cages. BMC Musculoskelet Disord. 2012;13:172.

30. Chou YC, Chen DC, Hsieh WA, et al. Efficacy of anterior cervical fusion: comparison of titanium cages, polyetheretherketone (PEEK) cages and autogenous bone grafts. J Clin Neurosci. 2008;15(11):1240-1245.
31. Li ZJ, Wang Y, Xu GJ, Tian P. Is PEEK cage better than titanium cage in anterior cervical discectomy and fusion surgery? A meta-analysis. BMC Musculoskelet Disord. 2016;17: 379.

32. Bocahut N, Audureau E, Poignard A, et al. Incidence and impact of implant subsidence after stand-alone lateral lumbar interbody fusion. Orthop Traumatol Surg Res. 2018;104(3): 405-410.

33. Chung RD, Hunter-Smith DJ, Spychal RT, et al. A systematic review of intraoperative process mapping in surgery. Gland Surg. 2017;6(6):715-725.

\section{Disclosures}

Dr. Campbell reports being a consultant to Stryker and Medtronic. Dr. Nunley reports receiving royalties from K2M, Zimmer Biomet, Camber Spine, and Integrity Spine; serving on the speakers bureau for K2M, Zimmer Biomet, Spineology, and Camber Spine; being a consultant for K2M, Zimmer Biomet, Spineology, Vertiflex, Camber Spine, Integrity, and Centinel Spine; and having stock options in Paradigm Spine, Spineology, and Camber Spine. Dr. Kerr reports being a consultant to Centinal Spine. Dr. Wadhwa reports being a consultant to Spineart. Dr. Stone reports being a consultant to Organogenesis and Spineology.

\section{Author Contributions}

Conception and design: all authors. Acquisition of data: Campbell, Cavanaugh, Stone. Analysis and interpretation of data: Campbell, Cavanaugh, Nunley, Utter, Kerr, Stone. Drafting the article: Campbell. Critically revising the article: all authors. Reviewed submitted version of manuscript: all authors. Approved the final version of the manuscript on behalf of all authors: Campbell. Statistical analysis: Campbell, Stone. Administrative/ technical/material support: Campbell, Stone. Study supervision: Campbell.

\section{Correspondence}

Peter G. Campbell: Spine Institute of Louisiana, Shreveport, LA. pcampbell@louisianaspine.org. 